\title{
Self-nanoemulsifying system improves oral absorption and enhances anti-acute myeloid leukemia activity of berberine
}

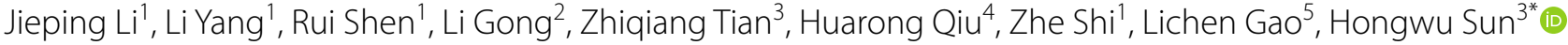
and Guangsen Zhang ${ }^{6^{*}}$

\begin{abstract}
Background: Recently, we found that berberine (BBR) exerts anti-acute myeloid leukemia activity, particularly toward high-risk and relapsed/refractory acute myeloid leukemia MV4-11 cells in vitro. However, the poor water solubility and low bioavailability observed with oral BBR administration has limited its clinical use. Therefore, we design and develop a novel oil-in-water self-nanoemulsifying system for BBR (BBR SNE) to improve oral bioavailability and enhance BBR efficacy against acute myeloid leukemia by greatly improving its solubility.

Results: This system (size $23.50 \pm 1.67 \mathrm{~nm}$, zeta potential $-3.35 \pm 0.03 \mathrm{mV}$ ) was prepared with RH40 (surfactant), 1,2-propanediol (co-surfactant), squalene (oil) and BBR using low-energy emulsification methods. The system loaded BBR successfully according to thermal gravimetric, differential scanning calorimetry, and Fourier transform infrared spectroscopy analyses. The release profile results showed that BBR SNE released BBR more slowly than BBR solution. The relative oral bioavailability of this novel system in rabbits was significantly enhanced by 3.41 -fold over that of BBR. Furthermore, Caco-2 cell monolayer transport studies showed that this system could help enhance permeation and prevent efflux of BBR. Importantly, mice with BBR SNE treatment had significantly longer survival time than BBRtreated mice $(P<0.001)$ in an MV4-11 engrafted leukemia murine model.
\end{abstract}

Conclusions: These studies confirmed that BBR SNE is a promising therapy for acute myeloid leukemia.

Keywords: Berberine, Self-nanoemulsifying system, Acute myeloid leukemia, MV4-11, Caco-2 monolayer transport

\section{Background}

Acute myeloid leukemia (AML) is the most common acute leukemia worldwide [1]. In patients diagnosed before 60 years of age, it is curable in $35-40 \%$ of cases. However, only $5-15 \%$ of those presenting with the disease later in life can be cured [2-5]. Almost half of patients reach complete remission, but approximately $10 \%$ of patients have a median survival of only 1 year. For more than four decades since the combination of an anthracycline (most often daunorubicin) and cytarabine

\footnotetext{
*Correspondence: sunhongwu2001@163.com; zgsllzy@163.com

${ }^{3}$ Army Military Medical University of Chinese PLA, Chongqing 400038,

People's Republic of China

${ }^{6}$ Department of Hematology, The Second Xiangya Hospital of Central South University, Changsha 410008, Hunan, People's Republic of China Full list of author information is available at the end of the article
}

was first used for standard therapy, the " $3+7$ " regimen has remained the standard therapy for AML [6]. The long term disease-free survival of AML patients under age 60 remains around 40\%, with minimal improvement over the past several decades. The chemotherapy effectiveness may have hit a ceiling for treating AML, especially for older patients and those who either tend to relapse or have intermediate- or high-risk factors associated with AML [7]. FMS-like tyrosine kinase 3 internal tandem duplication (FLT3-ITD) mutations is over-expressed in $20-30 \%$ of AML cases and the most common molecular alteration in AML [8]. Patients with FLT3-ITD-mutated acute myeloid leukaemia, particularly those with a high allelic frequency, relapse quickly and have a shortened overall survival compared with patients who have the wild-type FLT3 [9]. It is well 
known that it is notoriously hard to treat [10]. Thus, an novel effective therapeutic drugs for the patient of AML, especially FLT3-ITD mutation are vital.

Berberine (BBR), a quaternary proto-berberine isoquinoline alkaloid, is a well-known naturally occurring medicine derived from the root and the stem bark of numerous plants such as Hydrastis canadensis, Berberis aquifolium, Berberis aristata, Berberis vulgaris and many other plants [11]. BBR is widely used for gastrointestinal infections and various inflammations in Asian countries [12]. It has many pharmacological actions, including activity against tumors [13, 14], microbe [15], inflammation $[15,16]$, neurodegenerative disease $[17,18]$ and other diseases. Recent studies have found that BBR exerts cytotoxicity and inhibits telomerase and topoisomerase, promotes the proliferation apoptosis in human leukemia cells by specifically binding to oligonucleotides or polymorphic nucleic acid and by stabilizing DNA triplexes or G-quadruplexes; the electrostatic interactions may be quantified in terms of the Hill model of cooperative interactions [19-22]. BBR showed antiproliferative activity and promptly localized to the nucleus $5 \mathrm{~min}$ to $15 \mathrm{~min}$ after BBR treatment in HL-60 human promyelocytic leukemia cells [22]. And also, research reported that BBR significantly inhibited the viability of AML THP-1 cells without incurring cytotoxicity to normal monocytes, which suggests that BBR could be an effective therapeutic agent against AML leukemia cells [23].

Importantly, we recently found that BBR effectively inhibits MV4-11 cells, an FMS-like tyrosine kinase 3 (FLT3)-mutated human AML cell line that is resistant to the commonly used anti-leukemia drug cytarabine (AraC) [24]. Based on these data, BBR may provide a new strategy for AML treatment, especially FLT3-mutated AML. However, BBR has extremely poor oral bioavailability $(<1 \%)$ [25], because of its poor aqueous solubility, extensive intestinal elimination and hepatobiliary excretion, as well as the Pgp-mediated efflux and the multidrug efflux pump induced low intestinal permeability [25-29]. Therefore, it is very important to design a formulation that improves the water solubility and facilitates the oral bioavailability of BBR.

Self-nanoemulsifying systems serve as a means to improve the bio-safety of lipophilic compounds and to improve their solubility, membrane transport, and absorption via the lymphatic system by bypassing firstpass metabolism [30]. A higher solubilization capacity, rapid onset of action, and reduced inter-subject differences are the advantages of self-nanoemulsifying system over with conventional systems [31]. In recent years, this system has emerged as a promising new technique to increase solubility, reduce side effects and enhance anti-tumor capacity [32]. However, there has been no study concerning self-nanoemulsifying systems encapsulating BBR for treatment of relapsed/refractory AML.

In this study, a novel self-nanoemulsifying system of BBR was prepared using a low-energy emulsification method to greatly improve BBR solubility. Additionally, we studied the in vitro release and in vivo pharmacokinetic characteristics of this system and investigated the mechanism by which it increases BBR oral bioavailability by using a Caco-2 cell monolayer model. Importantly, the anti-leukemia activity of the system was evaluated in an MV4-11 xenograft mouse model.

\section{Methods \\ Materials}

MV4-11 and Jurkat cells were obtained from American Type Culture Collection (ATCC, Manassas, USA) and cultured in IMDM (HyClone, Thermo scientific, USA) at $37{ }^{\circ} \mathrm{C}$ with $5 \% \mathrm{CO}_{2}$. Caco-2 cells were obtained from the Biomedical Analysis Center, TMMU and were cultured in DMEM (HyClone, Thermo scientific, USA) at $37^{\circ} \mathrm{C}$ with $5 \% \mathrm{CO}_{2}$. All cells were frozen in liquid nitrogen in $90 \%$ FBS (HyClone, Thermo scientific, USA) and 10\% DMSO at $-196{ }^{\circ} \mathrm{C}$. Cells of passages $5-10$ were used in the study.

\section{In vitro anti-acute myeloid leukemia activity assay}

The anti-AML activity of BBR against Jurkat and MV4-11 cells was studied using MTT assays. Cells were seeded at $1 \times 10^{5} / \mathrm{mL}$ in 96 -well plates exposed to BBR suspension (Baoja Natural Plant Development Co. Ltd. Baoji, Shan'xi, China) at various concentrations $(10,20,50,100,150$, $200 \mu \mathrm{g} / \mathrm{mL}$ ) and $200 \mu \mathrm{g} / \mathrm{mL}$ cytosine arabinoside water solution (Ara-C, Pfizer, NY, USA) incubated after treatment for $24 \mathrm{~h}$. Absorption at $450 \mathrm{~nm}$ was measured using an iMark Microplate reader (Bio-Rad, USA) after added $10 \mu \mathrm{L} /$ cell of CCK-8. Cell viability was calculated: Cell viability $(\%)=\left(\mathrm{OD}_{\text {treatment }} / \mathrm{OD}_{\text {control }}\right) \times 100 \%$. Each experiment was performed in triplicate.

\section{System component determination}

The solubility of BBR in the oils (peanut, saxol and squalene), surfactants (Tween 80, Cremophor EL40, Cremophor RH40) and co-surfactants (ethanol, glycerin and propylene glycol), was investigated by adding excess BBR to these compounds. Then, the samples were mixed for $60 \mathrm{~s}$ and shaken for $24 \mathrm{~h}$ in a water bath at $37^{\circ} \mathrm{C}$ to reach equilibrium. Finally, the concentration of BBR in these mixtures was determined using high-performance liquid chromatography (HPLC, Agilent 1260; Symmetry C18 column [ $5 \mu \mathrm{m}, 4.6 \times 250 \mathrm{~mm}$ ]; flow phase: acetonitrile/0.03 $\mathrm{M}$ potassium dihydrogen phosphate solution $=40 / 60$; flow rate: $1.0 \mathrm{~mL} / \mathrm{min}$; detection wavelength: $263 \mathrm{~nm}$; injection volume: $10 \mu \mathrm{L}$ ). 


\section{Optimize drug additive determination of this system}

Based on a preliminary experiment, the system was composed of squalene (oil), RH40 (surfactant), and 1,2-propanediol (co-surfactant). The Smix surfactant/cosurfactant ratios were 3:1, 4:1 and 5:1. A series of Smix and oil ratios (1:9, 2:8, 3:7, 4:6, 5:5, 6:4, 7:3, 8:2 and 9:1) were added and then gently agitated until the samples had a transparent appearance. The physical state of the self-nanoemulsifying system is shown in a pseudo-phase diagram in which one axis represents the water, another axis represents the oil and a third axis represents the mixture of co-surfactant and surfactant; the diagram was prepared using Origin 7.0 software (Origin Lab Corporation, USA) [33, 34]. To determine the optimum BBR addition amount, four concentrations $(0.1 \%, 0.2 \%, 0.5 \%$, and $1 \%)$ were examined. These samples were prepared using previously described methods.

\section{Preparation of the self-nanoemulsifying system}

In general, Smix was prepared by mixing RH 40 and 1,2-propanediol at a 4:1 (w/w) ratio. Next, squalene was added to Smix ratio of 8:2 (Smix: squalene) and berberine. The mixture was added drop-wise under gentle agitation to the water phase. In addition, the mixtures were assessed in this system when they were at low viscosity and showed a clear appearance. Blank control (blank SNE) was obtained as described previously, except the drug was replaced with water.

\section{Morphological and physicochemical characteristics}

The ultra-structure of this system was observed using previously described methods [35]. All samples after dilution 50 times with water were examined with a JEM1230 transmission electron microscope (TEM, JEOL Ltd., Tokyo, Japan) at $120 \mathrm{kV}$ voltage after negative straining with $1 \%$ phosphotungstic acid solution ( $\mathrm{pH} 7.4)$. The morphology of the samples was subsequently characterized by JEM-6700F scanning electron microscopy (SEM, JEOL Ltd., Tokyo, Japan). Physicochemical characteristics, including size, polydispersity index, and zeta potential were determined with a Nano ZS 90 (Malvern Instruments Ltd., UK).

\section{Encapsulation efficiency and drug loading}

Encapsulation efficiency (EE) and drug loading (DL) of BBR SNE were investigated based on previously reported methods [33]. Briefly, $0.2 \mathrm{~mL}$ of blank SNE or BBR SNE was added to $0.4 \mathrm{~mL}$ of absolute ethanol. Then, the samples were centrifuged $(13,000 \times g, 30 \mathrm{~min})$ after mixing and shaking for $60 \mathrm{~s}$ to ensure complete emulsification. The precipitates were added to $0.3 \mathrm{~mL}$ of water after the supernatants were removed. The BBR content of the precipitates and supernatants was determined via HPLC. The following equations were used to calculate encapsulation efficiency (EE) and drug loading (DL): EE $\quad(\%)=($ Actual $/$ Theoretical drug loading $) \times 100 \%$. $\mathrm{DD}=$ Actual drug loading/sample volume.

\section{Physical state and drug interaction evaluation of this system}

The physical state and drug interaction of the system was evaluated via differential scanning calorimetry (DSC) and thermal gravimetric (TG) analysis based on the previously method [36]. The samples were examined using a Q600 instrument (TA Instruments, New Castle, USA) at a heating rate of $10^{\circ} \mathrm{C} / \mathrm{min}$ from room temperature up to $200{ }^{\circ} \mathrm{C}$ under a nitrogen atmosphere. The weight loss of BBR and BBR SNE were measured, and samples were carefully sealed in an aluminum plate. The FTIR absorption data of the suspension (BBR) and self-nanoemulsion delivery system (BBR SNE) samples after preparation using the $\mathrm{KBr}$ disk method were obtained using a Lambda 950 spectrometer (PerkinElmer, Boston, USA) with a resolution of $4 \mathrm{~cm}^{-1}$ at 64 scans in the range of $400-4000 \mathrm{~cm}^{-1}$. By monitoring the locations of bands and peak frequencies, all interactions and chemical changes were determined.

\section{Stability and release profile study of this system Stability study}

The stability study was adapted from previously described methods [37]. The stability of the system was evaluated by centrifugation (13,000 rpm for $30 \mathrm{~min})$. In addition, the appearance of the samples was observed after six cycles (one cycle stored at $4{ }^{\circ} \mathrm{C}$ and another at $25{ }^{\circ} \mathrm{C}$, both for $48 \mathrm{~h}$ ). The system was considered unstable if it showed various appearances, such as creaming, drug or phase separation, turbidity, de-emulsification or precipitation.

\section{In vitro release profile in $P B S$}

BBR SNE and BBR were studied using the dialysis bag diffusion technique in vitro. Briefly, 5 - $\mathrm{mL}$ samples $(5 \mathrm{mg} /$ $\mathrm{mL}$ ) were added to a dialysis bag (SP132574, MWCO 10,000 g/mol, Sangon Biotech, Shanghai, China) separately. Then, the samples were immersed in $200 \mathrm{~mL}$ of PBS $(0.1 \mathrm{M}, \mathrm{pH}=7.4)$ release media and mixed at $100 \mathrm{rpm}$. The concentrations of the samples were measured by HPLC after release at various times $(0.17,0.33$, $0.5,1,1.5,2,4,6,9,12,24,36$ and $48 \mathrm{~h}$ ) with previously describe detection condition. Each experiment was performed in triplicate. 


\section{In vivo pharmacokinetic studies}

Rabbits were obtained from the Second Xiangya Hospital of Central South University (Hunan, China) and were randomly divided into two groups (BBR and BBR SNE, $\mathrm{n}=6)$. Rabbit blood $(0.5 \mathrm{~mL})$ was collected via the contra lateral vena cava auricular vein at $0,0.17,0.33,0.5$, $0.75,1,2,4,8,12,18$ and $24 \mathrm{~h}$ after oral BBR equivalent administration at $50 \mathrm{mg} / \mathrm{kg}$, based on the previously reported dose [38]. Blood samples were immediately separated by centrifugation at $4000 \mathrm{rpm}$ for $10 \mathrm{~min}$ and stored at $-20{ }^{\circ} \mathrm{C}$. All samples were analyzed via HPLC. Pharmacokinetic parameters were estimated using the noncompartmental methods by drug and statistic (DAS) software.

\section{Caco-2 cell cytotoxicity and transport studies Caco- 2 cell cytotoxicity}

Caco-2 cells were seeded in 96-well plates at a density of $1 \times 10^{4}$ cells/well and cultured in an incubator at $37{ }^{\circ} \mathrm{C}$ under $5 \% \mathrm{CO}_{2}$. The cell medium was replaced with the suspension and the BBR system at a series of finally concentrations $(6.25,12.5,25,50,100,500 \mu \mathrm{g} / \mathrm{mL})$, and the cells were incubated for $24 \mathrm{~h}$. The Caco- 2 cell cytotoxicity of the samples was determined using a Bio 6.0 Microplate Reader at $490 \mathrm{~nm}$ via the same MTT method. Each experiment was performed in triplicate.

\section{Caco-2 cell monolayer model transport studies}

Cells $\left(1.5 \times 10^{5}\right.$ cells/well $)$ were added in 24 -well Transwell plates $\left(0.4 \mu \mathrm{m}\right.$, Corning, USA) at $37{ }^{\circ} \mathrm{C}$ in a $5 \%$ $\mathrm{CO}_{2}$ humidified atmosphere. The culture medium was replaced every 2 days for the 1 st week and every day thereafter. Cells were cultured for 16-21 days prior to the following cellular uptake experiments. The integrity of the cell monolayers was monitored by measuring transepithelial electrical resistance (TEER). Only when the TEER value was $>300 \Omega \mathrm{cm}^{2}$ were the cell monolayers used in subsequent studies.

We used $50 \mu \mathrm{g} / \mathrm{mL}$ verapamil and $25 \mu \mathrm{g} / \mathrm{mL}$ BBR or BBR SNE in HBSS. The experiment was performed in both the apical to basolateral (AP-BL) and basolateral to apical (BL-AP) direction. Briefly, $200 \mu \mathrm{L}$ of drug with or without verapamil was added to the AP side in the AP$\mathrm{BL}$ experiments, and $800 \mu \mathrm{L}$ HBSS was added to the $\mathrm{BL}$ side. For the BL-AP experiments, $800 \mu \mathrm{L}$ of drug solution with or without verapamil was added to the BL side, and $200 \mu \mathrm{L}$ of blank HBSS was added to the AP side of the monolayer as the acceptor phase. Then, $50 \mu \mathrm{L}$ of the samples were taken from the AP side at 15, 30, 60, 90, 120 and 180 min time points and equal volumes of blank HBSS were added after each withdrawal. The samples were immediately stored at $-80{ }^{\circ} \mathrm{C}$ for subsequent analysis. Finally, samples were analyzed via HPLC. The apparent permeability coefficient (Papp) and efflux ratio (ER) were determined using the following equations: $\operatorname{Papp}=\mathrm{dQ} / \mathrm{dt} /$ (A.C0); ER = Papp (BL-AP)/Papp (AP-BL), where dQ/ $\mathrm{dt}$ is the rate of permeability, $\mathrm{A}$ is the surface area of the filter membrane, and $\mathrm{CO}$ is the initial concentration of insulin in the apical chamber. Each experiment was performed in triplicate.

\section{Anti-tumor activity against the MV4-11 xenograft model in vivo}

Animal maintenance and experimental procedures were carried out in accordance with the National Institutes of Health Guidelines for the Use of Experimental Animals. The MV4-11 xenograft mouse model was prepared according to previously described methods [39, 40]. Briefly, Spleen cells $\left(5 \times 10^{6}\right.$ cells/mouse) from MV4-11 transplanted NSG mice were intravenously injected into NSG (female, 6 weeks, Beijing Vitalstar Biotechnology Co., Ltd) mice via tail vein. One week after leukemia cell injection, all mice were randomly divided into five groups (PBS control, Blank SNE control, BBR, Ara-C, BBR SNE, $\mathrm{n}=10)$. All animals were treated at the same dose of $50(\mathrm{mg} / \mathrm{kg}) /$ day for 14 days by oral administrated with PBS, Blank SNE, BBR, and BBR SNE except Ara-C was administrated by tail vein injection, referring to the effect dose of Ara-C in the MV4-11 model [41]. During 98 days of observation, the survival time and number of all mice were recorded.

\section{Statistical analysis}

All the data are expressed as the average \pm SD (standard deviation). Differences between two groups were tested using Student's $t$-test. Differences in all data were analyzed using Student's $t$-test with GraphPad Prism 5.0 software (San Diego, USA). Differences are expressed as *** $P<0.001, * * P<0.01$ or $* P<0.05$.

\section{Results and discussion}

Berberine exerted anti-acute myeloid leukemia activity in vitro

To investigate the in vitro anti-AML activity of BBR, an MTT assay was used, and the cytotoxicity was measured $24 \mathrm{~h}$ (Fig. 1a) and $48 \mathrm{~h}$ (Fig. 1b) after treatment. Figure $1 \mathrm{a}, \mathrm{b}$ shows the inhibition activity of BBR in MV4-11 cells. Cytarabine, a commonly used clinical anti-AML drug, was used as control. At a concentration of $10 \mu \mathrm{g} /$ mL, BBR showed no obvious cytotoxicity toward MV411 cells at 24 or $48 \mathrm{~h}$ after treatment. Similarly, $200 \mu \mathrm{g} /$ $\mathrm{mL}$ of cytarabine failed to decrease the viability of MV411 cells. However, when the concentration was higher than $20 \mu \mathrm{g} / \mathrm{mL}$, BBR significantly induced cell death in a dose-dependent manner. We also performed the same test in Jurkat and HL-60 cells, obtaining similar results 

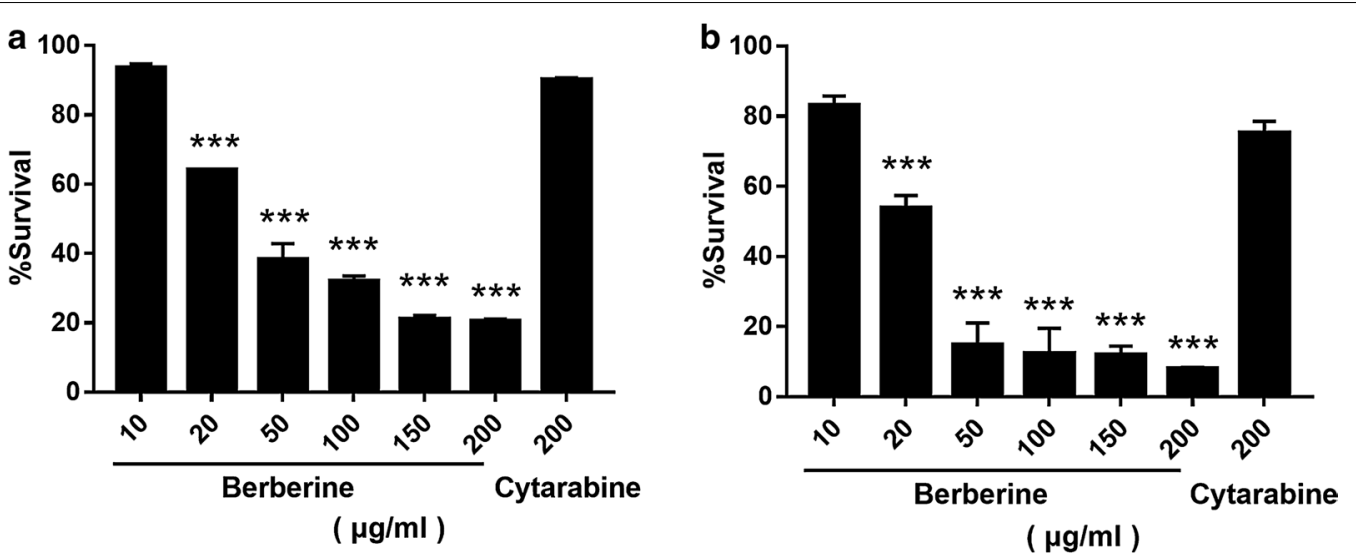

Fig. 1 Effect of a series of berberine and cytarabine solutions on the MV4-11 cell line at $\mathbf{a} 24 \mathrm{~h}$ and $\mathbf{b} 48 \mathrm{~h}$. The data are expressed as the mean \pm S.D. $(n=3) ;{ }^{* *} P<0.001,{ }^{* *} P<0.01$, and ${ }^{*} P<0.01$. Vs: compare with $10 \mu \mathrm{g} / \mathrm{mL}$ berberine

(Additional file 1: Figures S1A, S1B, S2A and S2B), with the only difference being that $200 \mu \mathrm{g} / \mathrm{mL}$ of cytarabine induced cell death in Jurkat and HL-60. Thus, the activity was approximately equal to $20 \mu \mathrm{g} / \mathrm{mL}$ BBR but was significantly weaker than that of $50 \mu \mathrm{g} / \mathrm{mL}$ BBR.

\section{Component determination of the system}

The solubility of BBR in Tween-80, RH40 and EL40 was $0.43 \pm 0.13 \mathrm{mg} / \mathrm{g}, 1.67 \pm 0.22 \mathrm{mg} / \mathrm{g}$, and $1.35 \pm 0.31 \mathrm{mg} / \mathrm{g}$, respectively. Additionally, the BBR solubility in the three co-surfactants 1,2-propanediol, glycerin and ethanol was $3.78 \pm 0.32 \mathrm{mg} / \mathrm{g}, 1.23 \pm 0.45 \mathrm{mg} / \mathrm{g}$, and $1.55 \pm 0.23 \mathrm{mg} / \mathrm{g}$, respectively. Furthermore, in the three oils (peanut, saxol and squalene), the solubility was $0.37 \pm 0.21 \mathrm{mg} / \mathrm{g}$, $0.43 \pm 0.12 \mathrm{mg} / \mathrm{g}$ and $2.13 \pm 0.55 \mathrm{mg} / \mathrm{g}$, respectively. Based on these solubility results, this nanoemulsifying system was prepared with RH-40, 1,2-propanediol, and squalene.

\section{Formation screening of the system}

Pseudo-ternary phase diagrams were prepared separately for various Smix ratios. The nanoemulsion areas were initially enlarged and then reduced with an increase in Smix (Fig. 2a-c). Additionally, the formation nanoemulsion area of Smix 4:1 (Fig. 2b) was demonstrated to be larger than that of the other Smix ratios (3:1, Fig. 2a; 5:1, Fig. 2c), which showed small and narrow areas. According to these pseudo-ternary phase diagrams, an Smix of RH-40/1,2-propanediol $=4: 1$, with the largest area, was the best formulation for the self-nanoemulsifying system.

Depending on the system loading with BBR, drug content could affect the nanoemulsion formation area focus. These areas first enlarged and then reduced with an increase in drug content (Fig. 3a-d). Additionally, the nanoemulsion formation area of $0.5 \%$ BBR SNE (Fig. 3c) was larger than that of the other contents $(0.1$, Fig. 3a; $0.2 \%$, Fig. $3 \mathrm{~b}$ and $1 \%$, Fig. $3 \mathrm{~d}$ ), all of which showed small and narrow nanoemulsion areas. Overall, a significant reduction in self-nanoemulsion area was evident only at $1 \%$ (Fig. 3d). Additionally, these results demonstrated that producing this system with high drug content $(>0.5 \%)$ does not confer an added stability benefit, a beneficial finding for industrial manufacture of self-nanoemulsifying products. Therefore, we identified the optimal drug content to be $0.5 \%$.

\section{Preparation and characterization of this system}

Based on the solubility and pseudo-ternary phase diagram results, a self-nanoemulsifying system (BBR SNE) with $0.5 \%$ BBR, 9.6\% RH-40, 2.4\% 1,2-propanediol, and $0.3 \%$ squalene was prepared using the phase inversion method. The BBR suspension was a suspended, turbid solution, while the novel system was a clarified, transparent solution. In this study, we prepared BBR SNE with 2.5 -fold improvement in water solubility compared with that of BBR $(2 \mathrm{mg} / \mathrm{mL})$. Figure 4 a shows that the mean size of these system particles was $23.50 \pm 1.67 \mathrm{~nm}$; $75 \%$ of the particles were smaller than $28.46 \mathrm{~nm}$, and $90 \%$ of the particles were smaller than $37.24 \mathrm{~nm}$. The PdI value was directly proportional to the particle size range. In this study, the PdI was $0.121 \pm 0.01$, which is less than 0.3 . This result suggests that the novel self-nanoemulsion delivery had a relatively limited particle-size distribution. As shown in Fig. 4b, the zeta potential was $4.17 \pm 0.82 \mathrm{mV}$. We next observed the morphology of this novel system using TEM (Fig. 4c) and SEM (Fig. 4d). Figure $4 \mathrm{c}, \mathrm{d}$ shows that most of the droplet particles were between 1 and $100 \mathrm{~nm}$, and the system was spherical when observed under both TEM and SEM. These results showed that this system had good characteristics and 
a
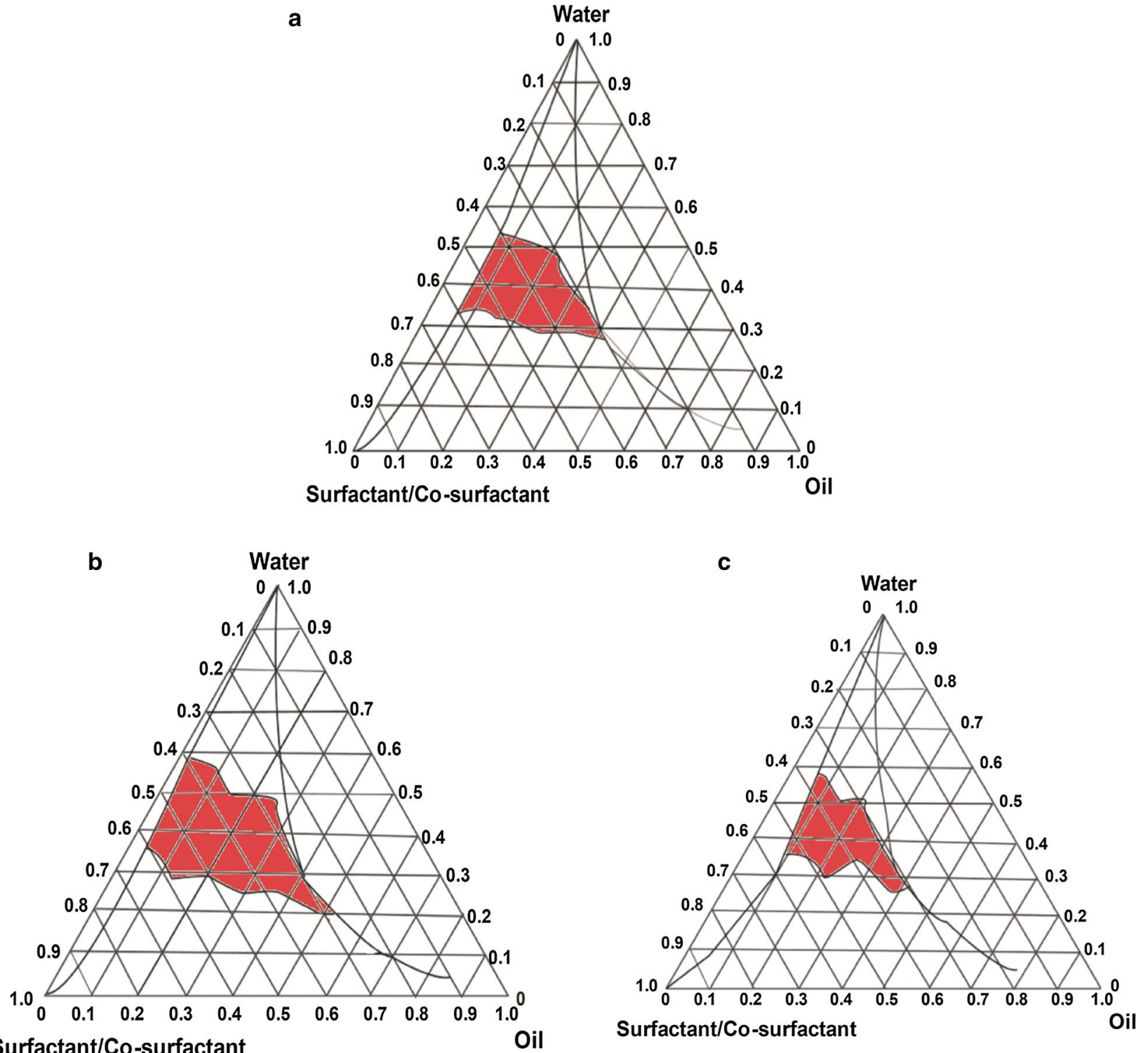

Surfactant/Co-surfactant

Fig. 2 Pseudo-ternary phase diagrams of the self-nanoemulsifying system composed of the following constituents: RH-40 (surfactant),

1,2-propanediol (co-surfactant), squalene (oil phase). a Surfactant and co/surfactant mixture ratio (Smix), 3:1; b Smix, 4:1; c Smix, 5:1. The red areas

represent the nanoemulsion formation regions

could fulfill the formation requirements. We detected the loading efficacy and drug loading amount of BBR SNE. We found that the loading efficacy and drug loading amount were $82.5 \pm 2.13 \%$ and $4321.23 \pm 3.33 \mu \mathrm{g} / \mathrm{mL}$, respectively.

\section{Evaluation of the physical state and drug interaction}

A characteristic single prominent peak was observed at $66.99{ }^{\circ} \mathrm{C}$ in the thermogram of the nanoemulsifying system that was absent in the thermogram of the BBR suspension (Fig. 5a). The DSC thermogram of the BBR suspension showed one peak at $74.79{ }^{\circ} \mathrm{C}$. We observed that this system lowered the melting point of the drug, suggesting a possible intracellular permeation mechanism in this system. One of the simplest interactions is hydrogen bonding between the drug chains, invoking hydrogen-bonded beta-structure interactions between the drug and system.

The temperature exhibiting the highest rate of weight loss was indicated by a peak in the thermogravimetric analysis data. The self-nanoemulsifying system and BBR suspension showed similar weight loss patterns with just 


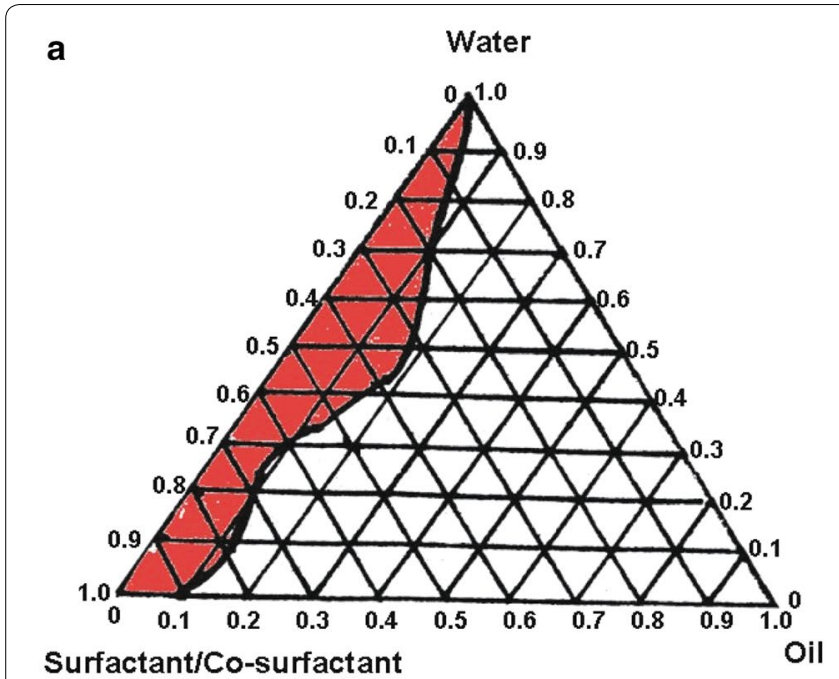

C

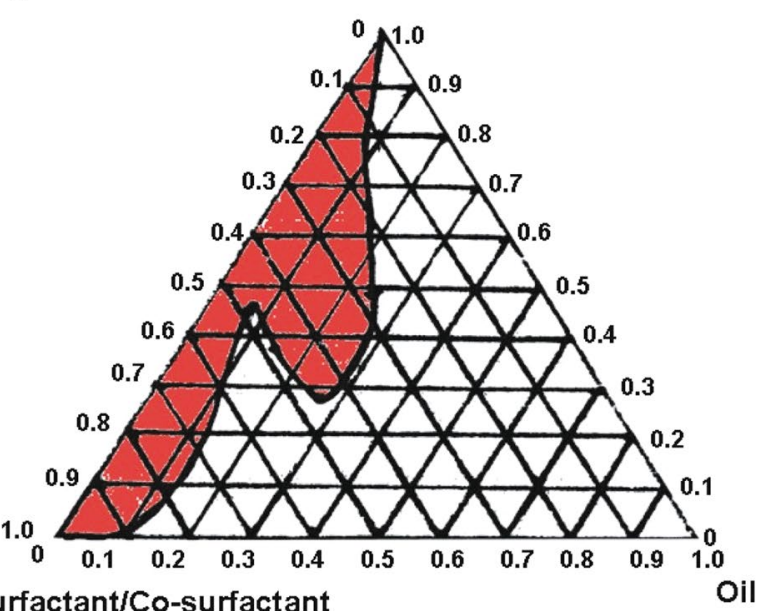

b

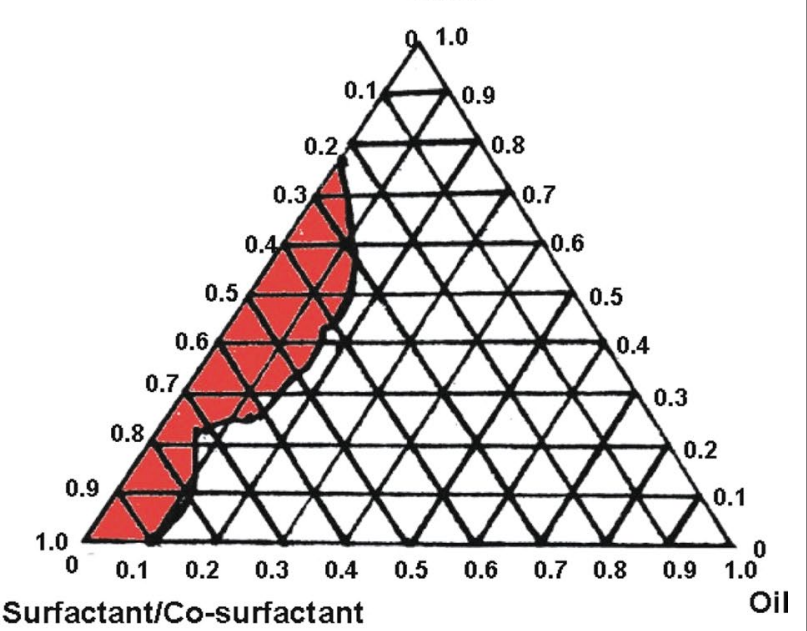

d

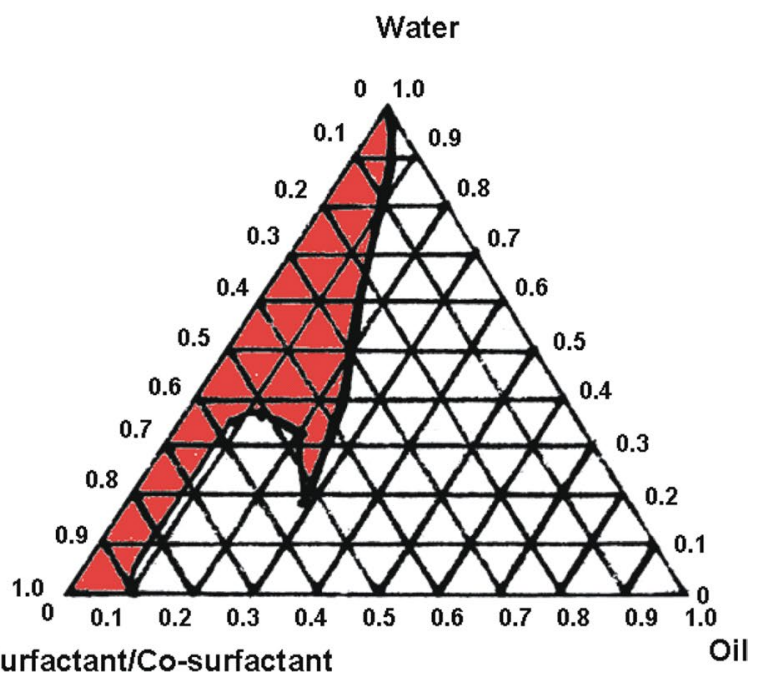

Fig. 3 Pseudo-ternary phase diagrams of the system (Smix=4:1) containing different berberine concentrations. a $0.1 \%$; b $0.2 \%$; c $0.5 \%$ and d $1.0 \%$ $(\mathrm{w} / \mathrm{w})$. The red areas represent the nanoemulsion formation regions

one step (Fig. 5b). The weight loss of this delivery began and ended at approximately 30 and $85.61{ }^{\circ} \mathrm{C}$, respectively. The weight loss of the BBR suspension began and ended at approximately 30 and $76.04{ }^{\circ} \mathrm{C}$, respectively. The thermogravimetric curve of the BBR suspension showed a weight loss of $0.28 \%$ within a specified temperature from 0 to $200{ }^{\circ} \mathrm{C}$ due to the evaporation of the water content of the self-nanoemulsion delivery system. Another decrease of $2.46 \%$ in the mass profile of this system occurred over a temperature range from 0 to $200{ }^{\circ} \mathrm{C}$. Mass transfer from a consistent viscous liquid into the gas state owing to the evaporation process is typically more difficult than that from a less viscous liquid.

FTIR spectra of the water solution and its system (Fig. 5d) indicated sharp peaks with proper intensities; vibration changes play a significant role in the intermolecular interactions in this system. In the FTIR spectra, the characteristic peaks were as follows: 700$1300 \mathrm{~cm}^{-1}$ (skeletal C-C vibrations), $1105.66 \mathrm{~cm}^{-1}$ (C-O), $1600.45 \mathrm{~cm}^{-1}$, and $1506.17 \mathrm{~cm}^{-1}$ aromatic $\mathrm{C}=\mathrm{C}$ stretching and skeleton vibration of aromatic $\mathrm{C}=\mathrm{C}$ ring stretching, $1390.31 \mathrm{~cm}^{-1}$ and $1364.75 \mathrm{~cm}^{-1}(C=C$ stretching), $1272.97 \mathrm{~cm}^{-1}$ (C-O-C stretching), and 1035.89-1142.01 $\mathrm{cm}^{-1}$ (in plane $=\mathrm{C}-\mathrm{H}$ bending). A characteristic carbonyl functional group peak at $1644 \mathrm{~cm}^{-1}$ along with the characteristic $\mathrm{C}-\mathrm{O}-\mathrm{C}$ stretching vibrations of the repeated $-\mathrm{OCH} 2 \mathrm{CH} 2$ chain of TPGS were observed in the region of 1104$1268 \mathrm{~cm}^{-1}$ (Fig. 5d). The absence of the characteristic peak of BBR at $1734 \mathrm{~cm}^{-1}$ confirmed entrapment in this 

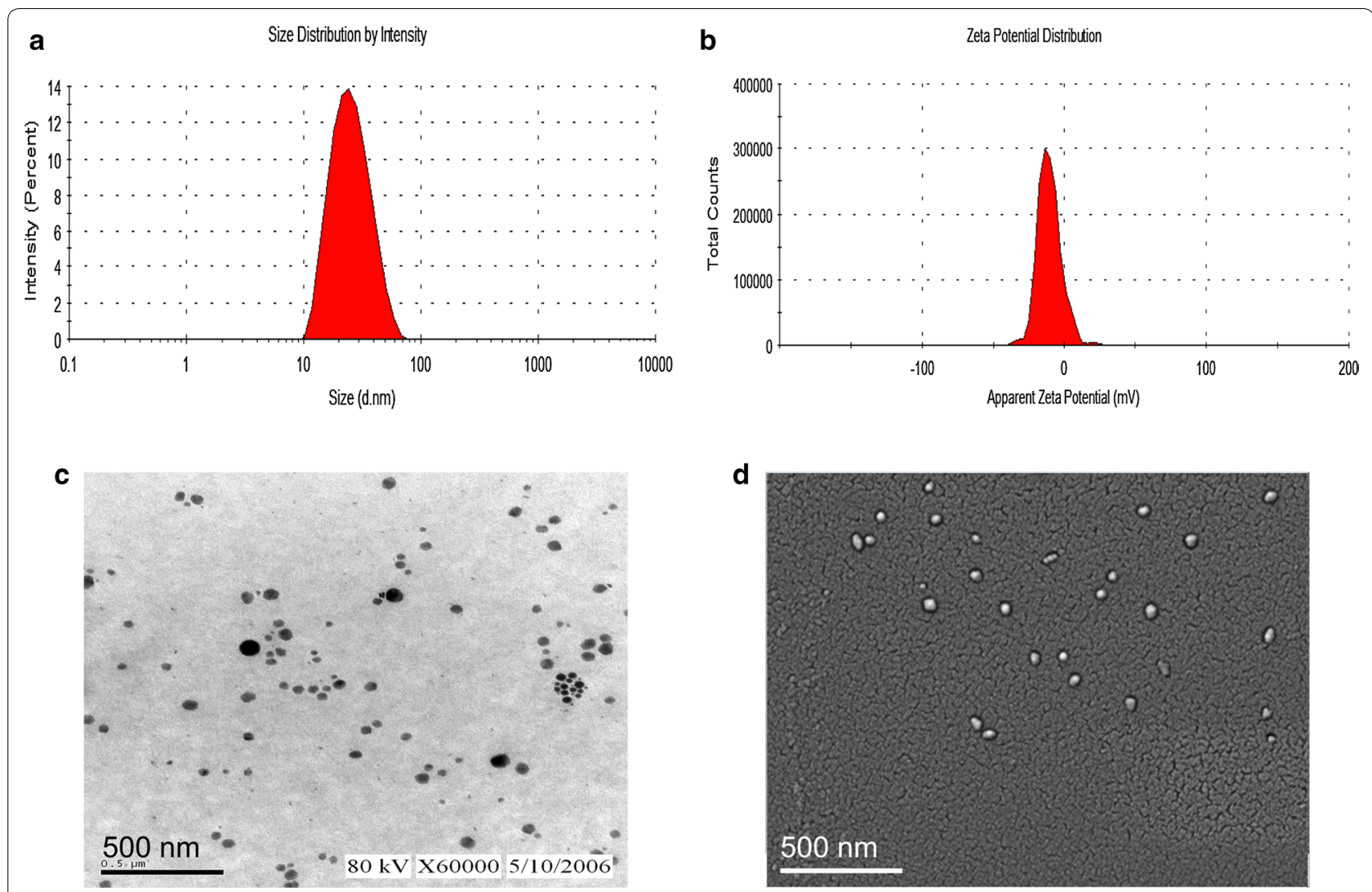

Fig. 4 Characteristics of the self-nanoemulsifying system. a Particle-size distribution; b zeta-potential distribution; c transmission electron micrograph and $\mathbf{d}$ scanning electron micrograph

system (Fig. 5d). These data showed that this system successfully loaded BBR.

\section{Stability and release profile study of the delivery system}

This novel system showed good thermodynamic and high centrifuge stability because it exhibited no creaming, de-emulsification, drug separation, turbidity precipitation, and/or phase separation after centrifugation at 13,000 rpm for $30 \mathrm{~min}$. These results demonstrated that the delivery system had good thermodynamic stability.

Release studies of the system and water solution were performed in PBS $(\mathrm{pH}=7.4)$. As shown in Fig. 6a, approximately $90 \%$ of BBR was released from suspension in $2.5 \mathrm{~h}$, while $90 \%$ of the drug was released from the system in $36 \mathrm{~h}$. Additionally, the near complete release $(90 \%$ release rate) time of the system was delayed 14.4-fold compared with that of the suspension. A possible reason for the delayed drug release from the system is that the drug was perhaps adsorbed on the surface and dissolved.

\section{In vivo pharmacokinetic studies}

The pharmacokinetic parameters of this novel system and the BBR suspension were compared. Figure 6b shows the plasma concentration profiles of BBR after oral administration of both formulations in rabbits. The pharmacokinetic parameters are summarized in Table 1 . The AUC of the system $(3639.94 \pm 10.37 \mu \mathrm{g} / \mathrm{mL} \mathrm{h})$ was 3.41-fold greater than that of the BBR suspension $(1071.26 \pm 6.15 \mu \mathrm{g} / \mathrm{mL} \mathrm{h})$, indicating $341 \%$ relative bioavailability of the system compared with the suspension formulation. The MRT was also significantly prolonged (1.52-fold) for the system $(3.85 \pm 0.11 \mathrm{~h})$ compared with the suspension $(2.54 \pm 0.12 \mathrm{~h})$. These results suggested that the system promoted absorption and sustained release of $\mathrm{BBR}$, thereby improving the relative bioavailability of BBR.

\section{Caco- 2 cell cytotoxicity}

A CCK-8 assay was conducted to assess the cytotoxicity of the system and BBR solution (Fig. 7a). The BBR SNE showed no significant cytotoxicity (cell viability $>90 \%$ ) at concentrations between 6.25 and $500 \mu \mathrm{g} / \mathrm{mL}$, while BBR showed no significant cytotoxicity at concentrations between 6.25 and $25 \mu \mathrm{g} / \mathrm{mL}$. Thus, a concentration of $25 \mu \mathrm{g} / \mathrm{mL}$ BBR and BBR SNE was selected for Caco- 2 cell monolayer transport studies. 


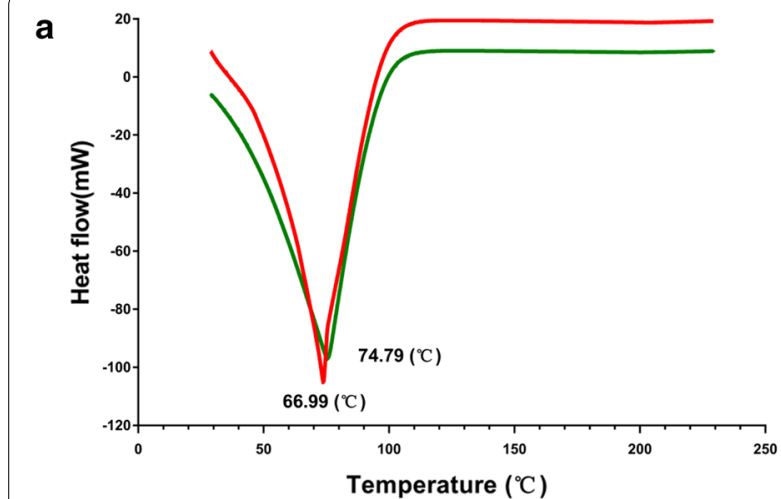

C

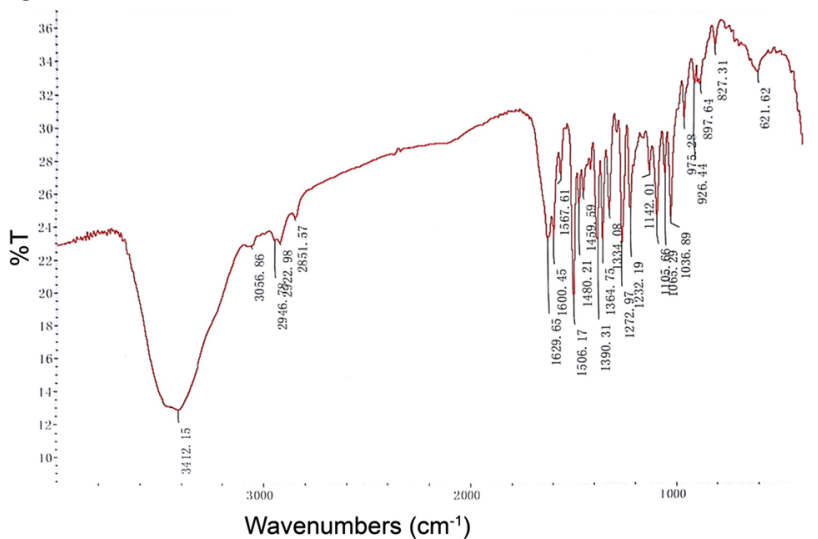

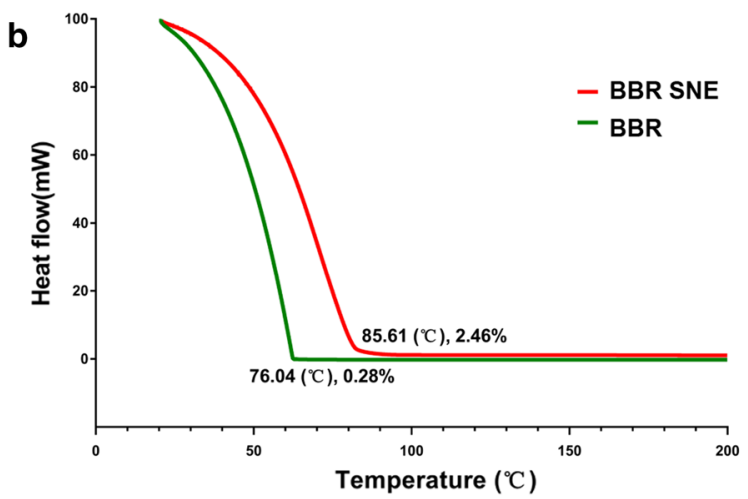

d

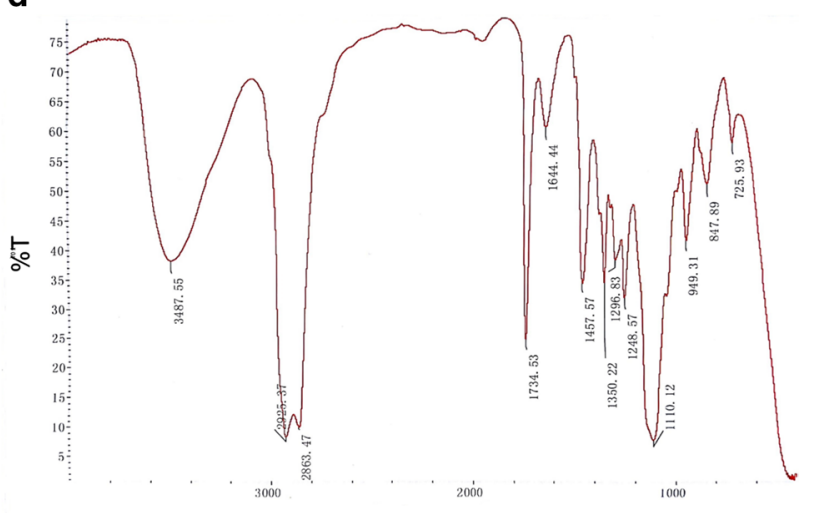

Wavenumbers $\left(\mathrm{cm}^{-1}\right)$

Fig. 5 Evaluation of the physical state and drug interaction of this system. a Differential scanning calorimetry; $\mathbf{b}$ thermogravimetric analysis; $\mathbf{c}$ FTIR spectrum of the berberine suspension; $\mathbf{d}$ FTIR spectrum of the system
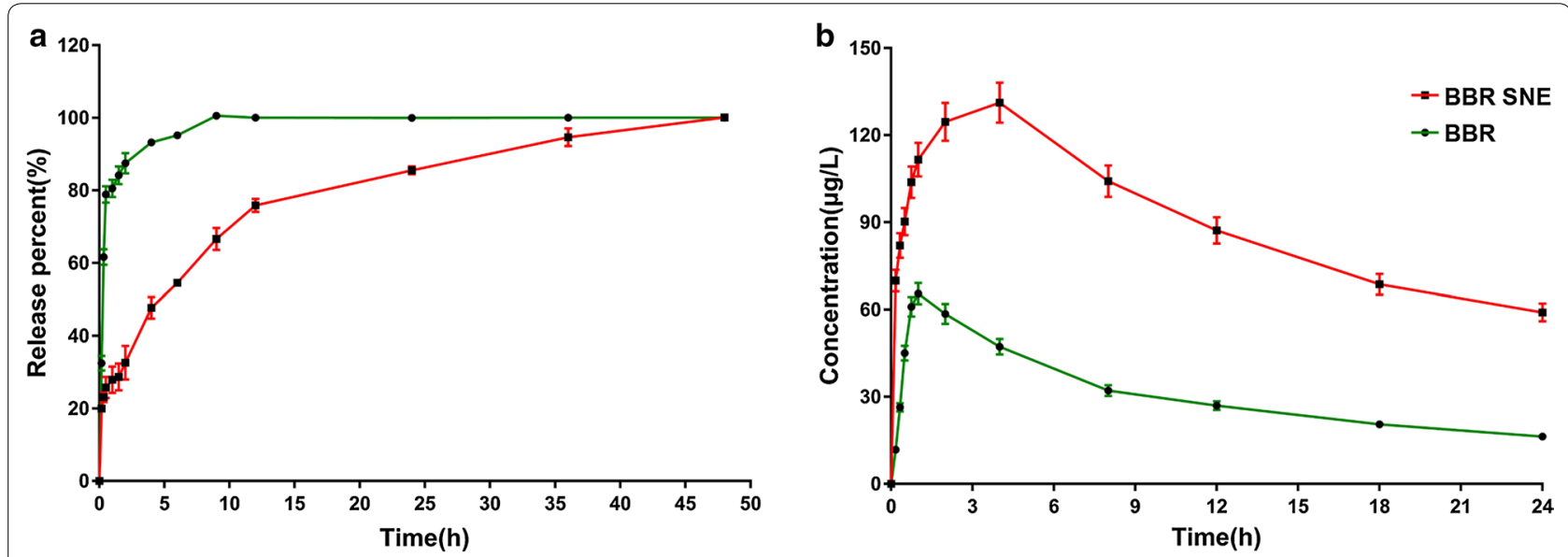

Fig. 6 In vitro release and in vivo pharmacokinetic studies. a In vitro release profile of the berberine suspension (BBR) and the system (BBR SNE) in PBS $(\mathrm{pH}=7.4)(n=3)$; b plasma concentration profiles of berberine after oral administration of BBR and BBR SNE in healthy rabbits $(n=6)$

\section{Caco-2 cell monolayer transport studies}

Experiments were performed in the apical to basolateral $(\mathrm{AP}-\mathrm{BL})$ and basolateral to apical (BL-AP) direction. As shown in Table 2, for the Caco-2 cell monolayer transport study (AP-BL), the absorptive concentration-time profiles of the BBR suspension 
Table 1 The pharmacokinetics parameters of berberine nanoemulsion in healthy rabbits

\begin{tabular}{llrc}
\hline Parameters & Unit & \multicolumn{1}{l}{$\begin{array}{l}\text { Berberine } \\
\text { suspension }\end{array}$} & \multicolumn{1}{l}{$\begin{array}{l}\text { Berberine } \\
\text { nanoeumlsion }\end{array}$} \\
\hline $\mathrm{AUC}_{0-24}$ & $\mu \mathrm{g} / \mathrm{mL} h$ & $1071.258 \pm 6.147$ & $3639.937 \pm 10.367^{* *}$ \\
$\mathrm{AUC}_{0-\infty}$ & $\mu \mathrm{g} / \mathrm{mLh}$ & $1263.456 \pm 1.472$ & $4319.261 \pm 26.784^{* * *}$ \\
$\mathrm{MRT}_{0-24}$ & $\mathrm{~h}$ & $12.195 \pm 2.581$ & $23.256 \pm 1.768^{* * *}$ \\
$\mathrm{MRT}_{0-\infty}$ & $\mathrm{h}$ & $16.722 \pm 1.732$ & $42.467 \pm 2.493^{* * *}$ \\
$\mathrm{Ka}$ & $1 / \mathrm{h}$ & $2.581 \pm 0.214$ & $0.710 \pm 0.369$ \\
$\mathrm{~T}_{\max }$ & $\mathrm{h}$ & $1.379 \pm 0.247$ & $4.205 \pm 0.234$ \\
$\mathrm{C}_{\max }$ & $\mu \mathrm{g} / \mathrm{mL}$ & $62.466 \pm 0.248$ & $113.699 \pm 0.369^{* *}$ \\
$\mathrm{t}_{1 / 2} \mathrm{a}$ & $\mathrm{h}$ & $1.065 \pm 0.231$ & $2.001 \pm 0.278^{* *}$ \\
$\mathrm{t}_{1 / 2} \beta$ & $\mathrm{h}$ & $16.649 \pm 0.321$ & $21.241 \pm 2.296^{* *}$ \\
$\mathrm{t}_{1 / 2} \mathrm{Ka}$ & $\mathrm{h}$ & $0.269 \pm 0.026$ & $0.976 \pm 0.325$ \\
\hline
\end{tabular}

Values are the mean $\pm S D, \mathrm{n}=6$

AUC Areas under the curve, $M R T$ mean retention time, $T_{\max }$ peak time, $C_{\max }$ pea concentration, $t_{1 / 2} a$ half-life of distribution, $t_{1 / 2} \beta$ half-life of elimination, $t_{1 / 2} K a$ half-life of absorbance

${ }^{* *} \mathrm{P}<0.01$, ${ }^{* * *} \mathrm{P}<0.001$, compared with berberine suspension

were too low to be detectable by HPLC. The efflux ratio of BBR SNE was $2.47 \pm 0.47$, suggesting the presence of an efflux transporter. In addition, the apparent permeability coefficient (Papp) values of BBR from A-to$B$ transport and from B-to-A efflux were summarized [27]. The permeability coefficients of BBR and BBR SNE with verapamil, a P-glycoprotein inhibitor, were significantly lower than with BBR, which indicated that BBR SNE reduced BBR efflux in Caco- 2 cell monolayers, and the drug was probably effluxed by P-glycoprotein.
Anti-tumor activity against an MV4-11 xenograft model in vivo

We evaluated the therapeutic performance of BBR SNE using the MV4-11 xenograft model. Mice were treated with the system and/or the suspension $(50 \mathrm{mg} / \mathrm{kg})$. Mice received PBS, blank SNE and cytarabine (Ara-C) as controls. Mice were orally administered the corresponding material within an experimental period of 14 days (Fig. 7b). The mice treated with the system or with Ara-C displayed higher survival rates $(70 / 30 \%$ at 98 days, respectively) than those treated with $\mathrm{BBR}, \mathrm{PBS}$ and blank SNE (no survival). These data suggested that treatment with this novel system markedly increased the survival rate of MV4-11 xenograft mice.

\section{Discussion}

In high income countries, over $90 \%$ of pediatric acute myeloid leukemia (AML) patients achieve complete remission with current intensive chemotherapy protocols. However, even with optimal therapies, 30-40\% of patients relapses and faces a dismal prognosis [42]. There are many research showed that FMS-like tyrosine kinase 3 (FLT3) internal tandem duplication (ITD) mutations, resulting in constitutive kinase activity in AML are associated with poor prognosis [43]. The complete response rate was found to be lower and the overall survival shorter than in non-FLT3 AML patients [44, 45]. To date, effective treatment regimens for FLT3 mutant AML patients remain lacking and represent an urgent need [8]. MV4-11 cell line possessing the FLT3 mutation had lower IC50s for azacitidine and panobinostat. In this study, for the first time, we showed that BBR had a strong
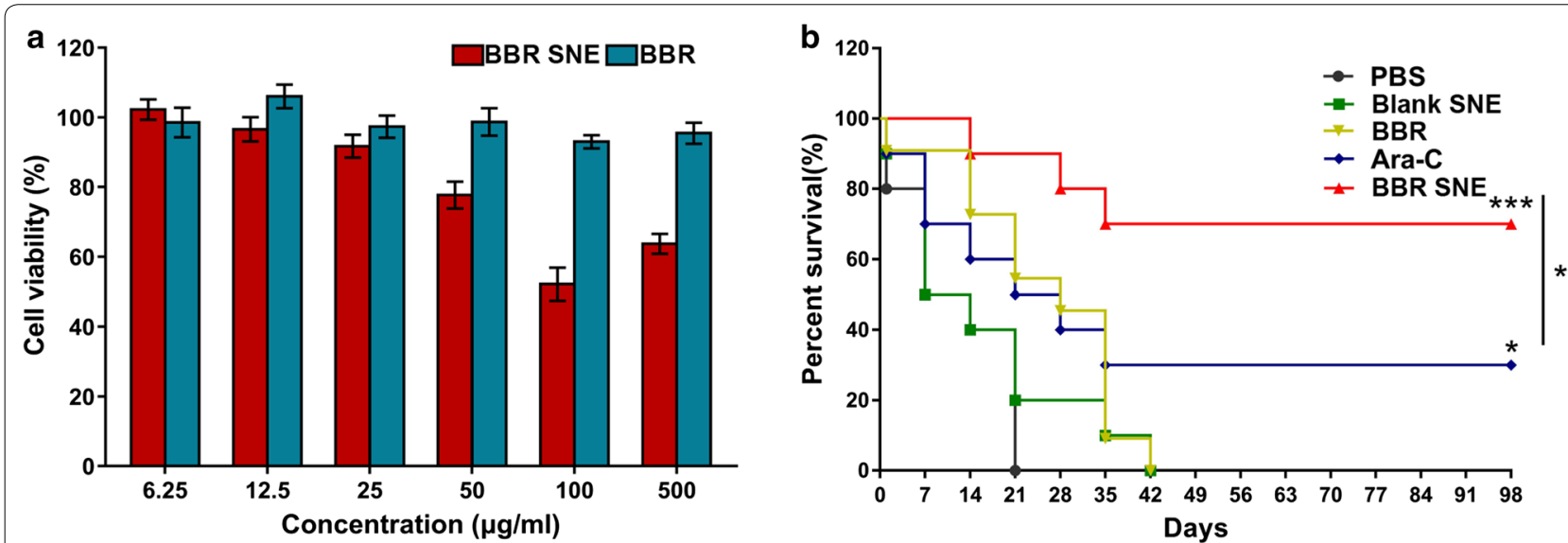

Fig. 7 Effect of cell viability and survival of the self-nanoemulsifying system. a Effect of berberine solution (BBR) and the system (BBR SNE) on Caco-2 cell viability as evaluated by MTT assays after treatment for $24 \mathrm{~h}$. The data are expressed as the mean \pm S.D. ( $\mathrm{n}=3$ ). $\mathbf{b}$ The survival rate of MV4-11 xenograft mice orally administered berberine suspension (BBR), PBS, Blank SNE and the system (BBR SNE) excepted for cytarabine (Ara-C) with the vein intravenous injection at 50 ( $\mathrm{mg} / \mathrm{kg}) /$ day for 14 consecutive days; the same volume of PBS and blank SNE were used as controls. The data are expressed as the mean \pm S.D. $(n=10)$; ${ }^{*} P<0.05$, and ${ }^{* * *} P<0.001$ 
Table 2 Apparent permeability coefficients (Papp) and efflux ratio (ER) of berberine and berberine SNE cross Caco-2 monolayer

\begin{tabular}{llll}
\hline Group & \multicolumn{2}{l}{ Papp $\left(\times \mathbf{1 0}^{-\mathbf{8}} \mathbf{c m} / \mathbf{s}\right)$} & ER \\
\cline { 2 - 3 } & AP-BL & BL-AP & \\
\hline Berberine & - & $2.771 \pm 0.213$ & - \\
Berberine SNE & $0.749 \pm 0.116$ & $1.852 \pm 0.271^{* *}$ & $2.473 \pm 0.467$ \\
Berberine SNE + Ver & $0.232 \pm 0.231^{*}$ & $1.325 \pm 0.172^{\#}$ & $5.711 \pm 0.524^{\# \#}$ \\
\hline
\end{tabular}

Values are the mean $\pm S D, n=3$

ER efflux ratio, Papp permeability coefficient, SNE self-nanoemulsifying system, Ver verapamil

${ }^{*} \mathrm{P}<0.05$, compared with berberine NE in AP-BL transport

** $P<0.01$, compared with berberine in BL-AP transport

\# $\mathrm{P}<0.01$, compared with berberine NE in BL-AP transport

\#\# $\mathrm{P}<0.01$, compared with berberine NE

cytotoxicity effect on the AML cell line MV4-11, which exhibits FLT3 mutations. And also, we had found that BBR significantly inhibit cell death in a dose-dependent manner in the MV4-11, Jurkat and HL-60. Hence, BBR is expected to be a new strategy for treat AML treatment, especially FLT3-mutated AML.

Berberine has been widely used as a broad-spectrum anti-microbial medicine for over 3000 years in China and used for wide clinical applications in Native American and Western medicine [46]. Recent studies have revealed that berberine can trigger autophagic cell death of tumor, and its beneficial effects are receded when the autophagic process is genetically or pharmacologically inactivated, suggesting that autophagy is indispensable for the protective effects of berberine [47]. Research reported berberine induced apoptosis in both HL-60 and WEHI- 3 cell lines in association with caspase-3 activity and mitochondria membrane potential depolarization [20]. The clinical use of berberine is largely limited because of its poor intestinal absorption and inadequate plasma level after oral administration [12]. Preclinical studies have shown that BBR has a very limited oral bioavailability (BA) $(<5 \%$ in plasma), largely due to its poor aqueous solubility, combined with low gastrointestinal absorption, and rapid metabolism [48]. However, novel self-nanoemulsion system is an emerging area of science and includes material application on the nanometre scale in various domains, including biology, chemistry, medicine, and engineering [49]. It is likely to improve the solubility stability and selectivity of drugs, improving the permeability of drugs while treating solid tumours through the enhanced permeability and retention effect, even for the blood brain barrier, which is high correlated with leukemia metastasis of the bone borrow [50]. There were a few reports focusing on the development of new dosage forms of BBR to increase its bioavailability, such as using the intestinal absorption enhancer, self-microemulsion, and solid lipid nanoparticles [51]. In our study, a novel self-nanoemulsion system was loaded $5 \mathrm{mg} / \mathrm{mL}$ berberine with size of $23.50 \pm 1.67 \mathrm{~nm}$ and zeta potential of $-3.35 \pm 0.03 \mathrm{mV}$ was designed using pseudo-ternary phase diagrams to greatly improve BBR solubility. After successful confirmation via TG, DSC, and FTIR analyses, the release profile results showed that this system released BBR more slowly than its solution. And also, the relative oral bioavailability of the system was significantly enhanced by 3.41-fold compared with BBR solution. And also, there is some reported that Berberine-loaded selenium-coated nanostructure lipid carriers (BB-SeNLCs) were more effective and significant in enhancing the bioavailability. The relative oral bioavailability of $663.65 \%$, respectively, compared with berberine solution [12]. Other study showed that the $\mathrm{O} / \mathrm{W}$ nanoemulsion of BBR showed a relative bioavailability of $440.40 \%$ compared with berberine solution. Our data is similar to these describe [27].

It has been reported that delivery of drugs which are P-gp substrates in nanoencapsulation form could be helpful for improvement of bioavailability through circumvention of P-gp efflux system [36]. The Caco-2 cell line is derived from a human colorectal carcinoma. It readily forms monolayers with morphological and functional similarities to the human small intestinal epithelium. In fact the FDA now recognizes the Caco- 2 cell monolayer as a viable model that replicates human intestinal absorption [52]. It does this not only because the cells form tight intercellular junctions similar to those of the intestinal epithelium but also because they express ATP-binding cassette $(\mathrm{ABC})$ membrane transporters such as $\mathrm{P}$-glycoprotein (P-gp) and multidrug resistance protein (MRP) that act to protect the body from toxic exogenous substances [53]. In our study, we found that the BA-LB (Papp, $\times 10^{-8} \mathrm{~cm} / \mathrm{s}$ ) of BBR SNE and BBR was $1.85 \pm 0.27$ and $2.77 \pm 0.21$, suggesting the presence of an efflux transporter. This data is also similar in the previous report [27].

The MV4-11 cell inoculated xenograft mouse model has been widely used to evaluate the therapeutic effect of FLT3-ITD-positive AML in vivo [54, 55]. In our study, the mice treated dose with the system or with Ara-C $50(\mathrm{mg} / \mathrm{kg}) /$ day. In the MV4-11 model, the group mice injected with $50(\mathrm{mg} / \mathrm{kg}) /$ day of cytarabine only had reduced tumor growth rates, and the tumor-growth inhibition was $61.94 \%$ on the 21 st day [41]. And also, there is reported that oral Berberine $200(\mathrm{mg} / \mathrm{kg} /$ day) for 3 weeks statistically significantly reduced the size and weight of spleen in these animals and also reduced the percentage of MAC-3 and CD11b cells in the blood [56]. We consider the dose of pharmacokinetic study of berberine and 
Ara-C efficacy dose of MV4-11 model. Our data suggest that oral treatment with this novel system significantly prolonged the survival of acute leukemia mice in the MV4-11 engrafted murine model. Of course, more and detail experiments such as other acute leukemia mice model and the mole action mechanism about BBR SNE will be further research. In shortly, in our study, these results demonstrated that a novel system loaded with poorly water-soluble berberine improved the aqueous solubility and oral absorption, and also effectively suppress MV-4-11 tumour growth and drastically increase mice survival rate in vivo.

\section{Conclusions}

We first found that BBR exerted anti-AML activity, especially toward high-risk and relapsed/refractory AML MV4-11 cells in vitro. Additionally, the novel self-nanoemulsifying system with a size of $23.50 \pm 1.67 \mathrm{~nm}$ and zeta potential of $-3.35 \pm 0.03 \mathrm{mV}$ was designed using pseudoternary phase diagrams to greatly improve BBR solubility. After successful confirmation via TG, DSC, and FTIR analyses, the release profile results showed that this system released BBR more slowly than its solution. The relative oral bioavailability of the system was significantly enhanced by 3.41-fold compared with BBR solution. Furthermore, the Caco-2 cell monolayer transport results showed that the system helped enhance permeation and prevented efflux of BBR. Importantly, we found that oral treatment with this novel system significantly prolonged the survival of acute leukemia mice in the MV411 engrafted murine model. These studies confirmed that this novel, efficient system is a promising therapy for acute myeloid leukemia.

\section{Additional file}

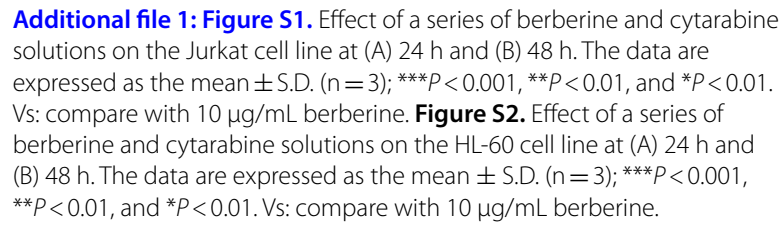
solutions on the Jurkat cell line at (A) $24 \mathrm{~h}$ and (B) $48 \mathrm{~h}$. The data are expressed as the mean \pm S.D. $(n=3)$; ${ }^{* *} P<0.001,{ }^{* *} P<0.01$, and ${ }^{*} P<0.01$. Vs: compare with $10 \mu \mathrm{g} / \mathrm{mL}$ berberine. Figure S2. Effect of a series of berberine and cytarabine solutions on the HL-60 cell line at (A) $24 \mathrm{~h}$ and (B) $48 \mathrm{~h}$. The data are expressed as the mean \pm S.D. $(n=3)$; ${ }^{* *} P<0.001$, ${ }^{*} P<0.01$, and ${ }^{*} P<0.01$. Vs: compare with $10 \mu \mathrm{g} / \mathrm{mL}$ berberine.

\section{Authors' contributions}

$J L, R S$ participated in the experiment and drew the scheme and figures; $J$, ZT, HQ and LG performed the experiments; JL, LY and LG wrote the paper. All authors contributed to the general discussion. All authors read and approved the final manuscript.

\footnotetext{
Author details

${ }^{1}$ Department of Hematology, Changsha Central Hospital, Changsha 410004, Hunan, People's Republic of China. ${ }^{2}$ Department of Clinical Laboratory, The Third Affiliated Hospital, Chongqing Medical University, Chongqing 401120, People's Republic of China. ${ }^{3}$ Army Military Medical University of Chinese PLA, Chongqing 400038, People's Republic of China. ${ }^{4}$ Air Force Military Medical
}

University of Chinese PLA, Xi'an 710000, Shanxi, People's Republic of China. ${ }^{5}$ Department of Pharmacy, Cancer Institute, Phase I Clinical Trial, Changsha Central Hospital, Changsha 410004, Hunan, People's Republic of China.

${ }^{6}$ Department of Hematology, The Second Xiangya Hospital of Central South University, Changsha 410008, Hunan, People's Republic of China.

\section{Acknowledgements \\ Not applicable.}

\section{Competing interests}

The authors declare that they have no competing interests.

Availability of data and materials

All dates generated and analyzed during this study are included in this paper.

Consent for publication

Not applicable.

Ethics approval and consent to participate

Compliance with ethical guidelines.

\section{Funding}

This work was supported by the National Natural Science Foundation of China under Grant No. 31670938; the Key Research and Development of Hunan Province under Grant No. 2016SK2066; the Natural Science Foundation Project Program of Chong Qing CSTC under Grant No. 2014jcyjA10107, and the Beijing Medical and Heath Found under Grant No. 20170701.

\section{Publisher's Note}

Springer Nature remains neutral with regard to jurisdictional claims in published maps and institutional affiliations.

Received: 10 May 2018 Accepted: 18 September 2018

Published online: 05 October 2018

\section{References}

1. Meyer SC, Levine RL. Translational implications of somatic genomics in acute myeloid leukaemia. Lancet Oncol. 2014;15(9):e382-94.

2. Hoseini SS, Cheung NK. Acute myeloid leukemia targets for bispecific antibodies. Blood Cancer J. 2017;7(2):e522.

3. Dohner H, Estey E, Grimwade D, et al. Diagnosis and management of AML in adults: 2017 ELN recommendations from an international expert panel. Blood. 2017;129(4):424-47.

4. Dohner H, Weisdorf DJ, Bloomfield CD. Acute myeloid leukemia. N Engl J Med. 2015;373(12):1136-52.

5. McNerney ME, Godley LA, Le Beau MM. Therapy-related myeloid neoplasms: when genetics and environment collide. Nat Rev Cancer. 2017;17(9):513-27.

6. Fan M, Li M, Gao L, et al. Chimeric antigen receptors for adoptive T cell therapy in acute myeloid leukemia. J Hematol Oncol. 2017;10(1):151.

7. Lim SH, Dubielecka PM, Raghunathan VM. Molecular targeting in acute myeloid leukemia. J Transl Med. 2017;15(1):183.

8. Zhi Y, Li B, Yao C, et al. Discovery of the selective and efficacious inhibitors of FLT3 mutations. Eur J Med Chem. 2018;155:303-15.

9. Stein EM. FLT3 inhibitors for relapsed or refractory acute myeloid leukaemia. Lancet Oncol. 2018;19(7):849-50.

10. Wang R, Li Y, Gong P, Gabrilove J, Waxman S, Jing Y. Arsenic trioxide and sorafenib induce synthetic lethality of FLT3-ITD acute myeloid leukemia cells. Mol Cancer Ther. 2018;17(9):1871-80.

11. Battu SK, Repka MA, Maddineni S, Chittiboyina AG, Avery MA, Majumdar S. Physicochemical characterization of berberine chloride: a perspective in the development of a solution dosage form for oral delivery. AAPS PharmSciTech. 2010;11(3):1466-75.

12. Yin J, Hou Y, Yin Y, Song X. Selenium-coated nanostructured lipid carriers used for oral delivery of berberine to accomplish a synergic hypoglycemic effect. Int J Nanomed. 2017;12:8671-80. 
13. Jabbarzadeh Kaboli $P$, Rahmat A, Ismail P, Ling KH. Targets and mechanisms of berberine, a natural drug with potential to treat cancer with special focus on breast cancer. Eur J Pharmacol. 2014;740:584-95.

14. Ortiz LM, Lombardi P, Tillhon M, Scovassi Al. Berberine, an epiphany against cancer. Molecules. 2014;19(8):12349-67.

15. Wen SQ, Jeyakkumar P, Avula SR, Zhang L, Zhou CH. Discovery of novel berberine imidazoles as safe antimicrobial agents by down regulating ROS generation. Bioorg Med Chem Lett. 2016;26(12):2768-73.

16. Lee $\mathrm{CH}$, Chen JC, Hsiang CY, Wu SL, Wu HC, Ho TY. Berberine suppresses inflammatory agents-induced interleukin-1 beta and tumor necrosis factor-alpha productions via the inhibition of IkappaB degradation in human lung cells. Pharmacol Res. 2007;56(3):193-201.

17. Ahmed T, Gilani AU, Abdollahi M, Daglia M, Nabavi SF, Nabavi SM. Berberine and neurodegeneration: a review of literature. Pharmacol Rep. 2015;67(5):970-9.

18. Ye M, Fu S, Pi R, He F. Neuropharmacological and pharmacokinetic properties of berberine: a review of recent research. J Pharm Pharmacol. 2009;61(7):831-7.

19. Abd El-Wahab AE, Ghareeb DA, Sarhan EE, Abu-Serie MM, El Demellawy MA. In vitro biological assessment of Berberis vulgaris and its active constituent, berberine: antioxidants, anti-acetylcholinesterase, anti-diabetic and anticancer effects. BMC Complement Altern Med. 2013;13:218.

20. Lin CC, Kao ST, Chen GW, Ho HC, Chung JG. Apoptosis of human leukemia HL-60 cells and murine leukemia WEHI-3 cells induced by berberine through the activation of caspase-3. Anticancer Res. 2006;26(1A):227-42.

21. Lin C-C, Lin S-Y, Chung J-G, Lin J-P, Chen G-W, Kao S-T. Down-regulation of cyclin B1 and up-regulation of Wee1 by berberine promotes entry of leukemia cells into the G2/M-phase of the cell cycle. Anticancer Res. 2006;26(2A):1097-104.

22. Okubo S, Uto T, Goto A, et al. Berberine induces apoptotic cell death via activation of caspase-3 and -8 in HL-60 human leukemia cells: nuclear localization and structure-activity relationships. Am J Chin Med. 2017:45(7):1497-511.

23. Mohammadi S, Seyedhoseini FS, Asadi J, Yazdani Y. Effects of berberine on the secretion of cytokines and expression of genes involved in cell cycle regulation in THP-1 monocytic cell line. Iran J Basic Med Sci. 2017;20(5):530-7.

24. Li P, Diab S, Yu M, et al. Inhibition of Mnk enhances apoptotic activity of cytarabine in acute myeloid leukemia cells. Oncotarget. 2016;7(35):56811-25.

25. Tan W, Li Y, Chen M, Wang Y. Berberine hydrochloride: anticancer activity and nanoparticulate delivery system. Int J Nanomed. 2011;6:1773-7.

26. Xiong W, Sang W, Linghu KG, et al. Dual-functional Brij-S20-modified nanocrystal formulation enhances the intestinal transport and oral bioavailability of berberine. Int J Nanomed. 2018;13:3781-93.

27. Li YJ, Hu XB, Lu XL, et al. Nanoemulsion-based delivery system for enhanced oral bioavailability and caco-2 cell monolayers permeability of berberine hydrochloride. Drug Deliv. 2017;24(1):1868-73.

28. Najar IA, Sachin BS, Sharma SC, Satti NK, Suri KA, Johri RK. Modulation of P-glycoprotein ATPase activity by some phytoconstituents. Phytother Res. 2010;24(3):454-8.

29. Qiu W, Jiang XH, Liu CX, Ju Y, Jin JX. Effect of berberine on the pharmacokinetics of substrates of CYP3A and P-gp. Phytother Res. 2009;23(11):1553-8.

30. Zhang Z, Qian H, Yang M, et al. Gambogic acid-loaded biomimetic nanoparticles in colorectal cancer treatment. Int J Nanomed. 2017;12:1593-605.

31. Onodera T, Kuriyama I, Andoh T, et al. Influence of particle size on the in vitro and in vivo anti-inflammatory and anti-allergic activities of a curcumin lipid nanoemulsion. Int J Mol Med. 2015;35(6):1720-8.

32. Ahmad G, El Sadda R, Botchkina G, Ojima I, Egan J, Amiji M. Nanoemulsion formulation of a novel taxoid DHA-SBT-1214 inhibits prostate cancer stem cell-induced tumor growth. Cancer Lett. 2017;406:71-80.

33. LiYF, Sun HW, Gao R, et al. Inhibited biofilm formation and improved antibacterial activity of a novel nanoemulsion against cariogenic Streptococcus mutans in vitro and in vivo. Int J Nanomed. 2015:10:447-62.

34. Sun H, Wei C, Liu B, et al. Induction of systemic and mucosal immunity against methicillin-resistant Staphylococcus aureus infection by a novel nanoemulsion adjuvant vaccine. Int J Nanomed. 2015;10:7275-90.

35. Cai W, Deng W, Yang H, Chen X, Jin F. A propofol microemulsion with low free propofol in the aqueous phase: formulation, physicochemical characterization, stability and pharmacokinetics. Int J Pharm. 2012;436(1-2):536-44.

36. Sahibzada MUK, Sadiq A, Faidah HS, et al. Berberine nanoparticles with enhanced in vitro bioavailability: characterization and antimicrobial activity. Drug Des Dev Ther. 2018;12:303-12.

37. Myc A, Kukowska-Latallo JF, Bielinska AU, et al. Development of immune response that protects mice from viral pneumonitis after a single intranasal immunization with influenza A virus and nanoemulsion. Vaccine. 2003:21(25-26):3801-14.

38. Feng R, Zhao ZX, Ma SR, Guo F, Wang Y, Jiang JD. Gut microbiota-regulated pharmacokinetics of berberine and active metabolites in beagle dogs after oral administration. Front Pharmacol. 2018;9:214.

39. Ranganathan $P, Y u X, N a C$, et al. Preclinical activity of a novel CRM1 inhibitor in acute myeloid leukemia. Blood. 2012;120(9):1765-73.

40. Ranganathan P, Kashyap T, Yu X, et al. XPO1 inhibition using selinexor synergizes with chemotherapy in acute myeloid leukemia by targeting DNA repair and restoring topoisomerase Ilalpha to the nucleus. Clin Cancer Res. 2016;22(24):6142-52.

41. Wang Y, Zhi Y, Jin Q, et al. Discovery of 4-((7H-Pyrrolo[2,3-d]pyrimidin-4-yl) amino)- $\mathrm{N}$-(4-((4-methylpiperazin-1-yl)methyl)p henyl)-1 $\mathrm{H}$-pyrazole-3-carboxamide (FN-1501), an FLT3- and CDK-kinase inhibitor with potentially high efficiency against acute myelocytic leukemia. J Med Chem. 2018;61(4):1499-518.

42. Bachas C, Schuurhuis GJ, Zwaan CM, et al. Gene expression profiles associated with pediatric relapsed AML. PLOS ONE. 2015;10(4):e0121730.

43. Kiyoi H, Yanada M, Ozekia K. Clinical significance of FLT3 in leukemia. Int J Hematol. 2005:82(2):85-92.

44. Thiede C, Steudel C, Mohr B, et al. Analysis of FLT3-activating mutations in 979 patients with acute myelogenous leukemia: association with FAB subtypes and identification of subgroups with poor prognosis. Blood. 2002;99(12):4326-35.

45. Ravandi F, Kantarjian H, Faderl S, et al. Outcome of patients with FLT3-mutated acute myeloid leukemia in first relapse. Leuk Res. 2010;34(6):752-6.

46. Wang S, Yu J, Suvira M, Setlow P, Li YQ. Uptake of and resistance to the antibiotic berberine by individual dormant, germinating and outgrowing Bacillus spores as monitored by laser tweezers Raman spectroscopy. PLoS ONE. 2015;10(12):e0144183.

47. La X, Zhang L, Li Z, Yang P, Wang Y. Berberine-induced autophagic cell death by elevating GRP78 levels in cancer cells. Oncotarget. 2017;8(13):20909-24

48. Shen R, Kim JJ, Yao M, Elbayoumi TA. Development and evaluation of vitamin E d-alpha-tocopheryl polyethylene glycol 1000 succinate-mixed polymeric phospholipid micelles of berberine as an anticancer nanopharmaceutical. Int J Nanomed. 2016;11:1687-700.

49. Hak S, Garaiova Z, Olsen LT, Nilsen AM, de Lange Davies C. The effects of oil-in-water nanoemulsion polyethylene glycol surface density on intracellular stability, pharmacokinetics, and biodistribution in tumor bearing mice. Pharm Res. 2015:32(4):1475-85.

50. Winter E, Pizzol CD, Locatelli C, et al. In vitro and in vivo effects of free and chalcones-loaded nanoemulsions: insights and challenges in targeted cancer chemotherapies. Int J Environ Res Public Health. 2014;11(10):10016-35.

51. Wang Z, Wu J, Zhou Q, Wang Y, Chen T. Berberine nanosuspension enhances hypoglycemic efficacy on streptozotocin induced diabetic C57BL/6 mice. Evid Based Complement Alternat Med. 2015;2015:239749.

52. Chen L, Lu X, Liang X, et al. Mechanistic studies of the transport of peimine in the Caco-2 cell model. Acta Pharm Sin B. 2016;6(2):125-31.

53. Planas JM, Alfaras I, Colom H, Juan ME. The bioavailability and distribution of trans-resveratrol are constrained by $A B C$ transporters. Arch Biochem Biophys. 2012;527(2):67-73

54. Gopalakrishnapillai A, Kolb EA, McCahan SM, Barwe SP. Epigenetic drug combination induces remission in mouse xenograft models of pediatric acute myeloid leukemia. Leuk Res. 2017;58:91-7.

55. Lopes de Menezes DE, Peng J, Garrett EN, et al. CHIR-258: a potent inhibitor of FLT3 kinase in experimental tumor xenograft models of human acute myelogenous leukemia. Clin Cancer Res. 2005;11(14):5281-91.

56. Yu FS, Yang JS, Lin HJ, et al. Berberine inhibits WEHI-3 leukemia cells in vivo. In Vivo. 2007:21(2):407-12. 